\title{
Phase shift induces currents in a periodic tube
}

\author{
Bao-quan $\mathrm{Ai} * *$ and Liang-gang $\mathrm{Liu}^{b}$ \\ a Institute for Condensed Matter Physics, \\ School of Physics and Telecommunication Engineering, \\ South China Normal University, 510006 GuangZhou, China. \\ $b$ The Faculty of Science Technology , \\ Macau University of Science and Technology, Macao.
}

(Dated: October 18, 2018)

\begin{abstract}
The average current of an overdamped Brownian particle moving along the axis of a threedimensional periodic tube is investigated in the presence of a symmetric potential and a temporally symmetric unbiased external force. Reduction of the spatial dimensionality from two or three physical dimensions to an effective one dimensional system entails the appearance of not only an entropic barrier but also an effective diffusion coefficient. We find that the phase shift between the tube shape and the potential can break the symmetry of the effective potential and can induce net currents. Under optimal condition, the current as a function of temperature and the phase shift possesses many extrema of alternating signs. The current may reverse its direction several times when temperature or the phase shift is changed. Our model is to describe the movement of molecular motors along microtubule.
\end{abstract}

PACS numbers: 05. 60. Cd, 05. 40. Jc, 87. 10. +e

Keywords: Phase shift, molecular motors, Current reversal 


\section{INTRODUCTION}

Molecular motors are protein molecules that can convert chemical energy, usually in the form of adenosine triphosphate (ATP), into mechanical forces and motion. Most organisms have many different motors that are specialized for particular purposes such as cell division, cell crawling, cell shape maintenance and movements of internal organelles $\frac{1,2}{2}$. Three different linear families of molecular motors have been identified $\underline{3}$ : Kinesins and dyneins that move along microtubule, myosins that move along actin filaments. From a theoretical point of view, molecular motors are microscopic objects that move unidirectionally along periodic structures. The problem of this unidirectionality belongs to a larger class of such problems involving rectifying processes at small scale. Noise-induced transport phenomena play a crucial role in molecular motors ${ }^{4} \underline{-8}$. A molecular motor is usually described by a ratchet system which is generally defined as a system that is able to transport particles in a periodic structure with nonzero macroscopic velocity in the absence of macroscopic force on average.

In these systems, directed Brownian motion of particles is generated by nonequilibrium noises in the absence of any net macroscopic forces and potential gradients. Typical examples are rocking ${\text { ratchets }{ }^{7}, \frac{8}{}}$, flashing ratchets $^{9}$, diffusion ratchets ${ }^{10}$, correlation ratchets $^{7,8}$ and white-shot-noise ratchets $^{5}$. In all these studies, the potential is asymmetric in space. It has shown that a unidirectional current can also appear for spatially symmetric potentials if the external random force is either asymmetric or spatially dependent. If spatially periodic structures are exposed to an additive Poissonian white shot noise, a macroscopic current occurs even in the absence of spatial asymmetry ${ }^{\underline{5}}$.

Most studies have revolved around the energy barrier. The nature of the barrier depends on which thermodynamic potential (internal energy or Helmholtz free energy) varies when passing from one well to the other, and its presence plays an important role in the dynamics of the system. Whereas energy barriers are more frequent in problem of solid-state physics (metals and semiconductors, coupled Josephson junction and photon crystal), entropy barriers are often encountered in soft-condensed matter and biological systems. Entropy barriers may appear when coarsening the description of a complex system in order to simplify its dynamics. Reguera and co-workers $\frac{12}{2}$ used the mesoscopic nonequilibrium thermodynamics 
theory to derive the general kinetic equation of the motor system and analyzed in detail the case of diffusion in a domain of irregular geometry in which the presence of the boundaries induces an entropy barrier when approaching the dynamics by a coarsening of the description. In their recent work ${ }^{13}$, they studied the current and the diffusion of a Brownian particle moving in a symmetric channel with a biased external force. They found that temperature dictates the strength of the entropic potential, and thus an increasing of temperature leads to a reduction of the current.

The focus of previous works on current were limited to the case of energy barriers in the presence of asymmetry in space or time. The present work is extended to the study to case of entropic barriers in the presence symmetry in space and time. We emphasize on finding how phase shift between the tube shape and the potential can induce a net current. Our model is to describe the movement of kinesins and dyneins along microtubule.

\section{THE CURRENT INDUCED BY A PHASE SHIFT}

Phenomena of current reversals may be of interest in molecular motors $\stackrel{14}{ }$. kinesins and dyneins move along tubulin filaments towards their plus and minus extremities, respectively. It is well known that the two current reversals effect allows one pair of motor proteins (two members)to move simultaneously in opposite directions along the microtubule inside the eukaryotic cells. One member of the pair moves one way along the tube while the other member of the pair moves in the opposite direction. In order to describe the movement of kinesins and dyneins along tubulin, a periodic tube model is proposed. We consider a Brownian particle moving in a symmetric periodic tube [Fig. 1] in the presence of a temporally symmetric unbiased external force. Its overdamped dynamics is described by the following Langevin equations written in a dimensionless form ${ }^{12,13}$,

$$
\begin{gathered}
\eta \frac{d x}{d t}=-\frac{\partial U(x)}{\partial x}+F(t)+\sqrt{\eta k_{B} T} \xi_{x}(t), \\
\eta \frac{d y}{d t}=\sqrt{\eta k_{B} T} \xi_{y}(t), \\
\eta \frac{d z}{d t}=\sqrt{\eta k_{B} T} \xi_{z}(t),
\end{gathered}
$$

where $x, y, z$ are the three-dimensional (3D) coordinates, $\eta$ is the friction coefficient of the particle, $k_{B}$ is the Boltzmann constant, $T$ is the absolute temperature and $\xi_{x, y, z}(t)$ 
is the Gaussian white noise with zero mean and correlation function: $<\xi_{i}(t) \xi_{j}\left(t^{\prime}\right)>=$ $2 \delta_{i, j} \delta\left(t-t^{\prime}\right)$ for $i, j=x, y, z .<\ldots>$ denotes an ensemble average over the distribution of noise. $\delta(t)$ is the Dirac delta function. Imposing reflecting boundary conditions in the transverse direction ensures the confinement of the dynamics within the tube, while periodic boundary conditions are enforced along the longitudinal direction for the reasons noted above. $F(t)$ is a temporally symmetric unbiased external force along the $x$ direction which is imparted as the result of energy gained via ATP hydrolysis and satisfies ${ }^{5-\underline{8}}$

$$
F(t)= \begin{cases}F_{0}, & n \tau \leq t<n \tau+\frac{1}{2} \tau \\ -F_{0}, & n \tau+\frac{1}{2} \tau<t \leq(n+1) \tau\end{cases}
$$

where $\tau$ is the period of the unbiased force and $F_{0}$ is its magnitude.

$U(x)$ is a symmetric potential with periodicity $L$ which is from the track of molecular motors [Fig. 1],

$$
U(x)=Q \sin \left(\frac{2 \pi x}{L}\right)
$$

where $Q$ is the amplitude of the potential. The shape of the tube (describing the structure of molecular motors) is described by its radius,

$$
\omega(x)=a \sin \left(\frac{2 \pi x}{L}+\phi\right)+b
$$

where $a$ is the parameter that controls the slope of the tube, $\phi$ is the relative phase shift between $\omega(x)$ and $U(x)$. The radius at the bottleneck is $b-a$.

The movement equation of a Brownian particle moving along the axis of the 3D (or 2D) tube can be described by the Fick-Jacobs equation $12,13,15,16$ which is derived from the 3D (or 2D) Smoluchowski equation after elimination of $y$ and $z$ coordinates by assuming equilibrium in the orthogonal directions. Reduction of the spatial dimensionality from two or three physical dimensions to an effective one dimensional system may involve not only the appearance of an entropic barrier, but also the effective diffusion coefficient. When $\left|\omega^{\prime}(x)\right|<<1$, the effective diffusion coefficient is 12,13

$$
D(x)=\frac{D_{0}}{\left[1+\omega^{\prime}(x)^{2}\right]^{\alpha}},
$$

where $D_{0}=k_{B} T / \eta$ and $\alpha=1 / 3$ and $1 / 2$ for two and three dimensions, respectively. The prime stands for the derivative with respect to the space variable $x$. 
Consider the effective diffusion coefficient and the entropic barrier, the dynamics of a Brownian particle moving along the axis of the 3D (or 2D) tube can be described by $\underline{12}, \underline{13,15}, 16$

$$
\frac{\partial P(x, t)}{\partial t}=\frac{\partial}{\partial x}\left[D(x) \frac{\partial P(x, t)}{\partial x}+\frac{D(x)}{k_{B} T} \frac{\partial A(x, t)}{\partial x} P(x, t)\right]=-\frac{\partial j(x, t)}{\partial x},
$$

where we define a free energy $A(x, t):=E-T S=U(x)-F(t) x-T k_{B} \ln h(x)$, here $E=U(x)-F(t) x$ is the energy, $S=k_{B} \ln h(x)$ is the entropy, $h(x)$ is the dimensionless width $2 \omega(x) / L$ in two dimensions, and the dimensionless transverse cross section $\pi[\omega(x) / L]^{2}$ of the tube in three dimensions. Here, we define a effective potential along the $x$ coordinate,

$$
U_{e f f}(x)=U(x)-T k_{B} \ln h(x) .
$$

$j(x, t)$ is the probability current density. $P(x, t)$ is the probability density for the particle at position $x$ and at time $t$. It satisfies the normalization condition $\int_{0}^{L} P(x, t) d x=1$ and the periodicity condition $P(x, t)=P(x+L, t)$.

If $F(t)$ changes very slowly with respect to $t$, namely, its period is longer than any other time scale of the system, there exists a quasisteady state. In this case, by following the method in $\underline{\underline{4}-13}$, we can obtain the current

$$
j(F(t))=\frac{k_{B} T\left[1-\exp \left(-\frac{F(t) L}{k_{B} T}\right)\right]}{\int_{0}^{L} h(x) \exp \left[\frac{-U(x)+F(t) x}{k_{B} T}\right] d x \int_{x}^{x+L}\left[1+\omega^{\prime}(y)^{2}\right]^{\alpha} h^{-1}(y) \exp \left[\frac{U(y)-F(t) y}{k_{B} T}\right] d y} .
$$

For the given external force (Eq. (4)), the average current ${ }^{7.8}$ is

$$
J=\frac{1}{\tau} \int_{0}^{\tau} j(F(t)) d t=\frac{1}{2}\left[j\left(F_{0}\right)+j\left(-F_{0}\right)\right]
$$

\section{RESULTS AND DISCUSSIONS}

Because the results from two and three dimensions are very similar, for the convenience of physical discussion, we now mainly investigate the current in three dimensions with $k_{B}=1$, $\eta=1$ and $L=2 \pi$. The results are shown in Figs. 2-5.

Figure 2 shows the current contours on $T-\phi$ plane. There are two hills and two vales in the plot. The current is negative in area A and C, positive in area B and D. In our model, the potential and the tube shape in space and the external force in time are symmetric. It is obvious that the phase shift between the potential and the tube shape leads to a net current. However, the current is always zero at $\phi=0, \phi$ and $2 \pi$. When $0<\phi<\phi_{a}$ and 
$\phi_{b}<\phi<2 \pi$, the particle will undergo a hill and a vale on increasing temperature, namely, the current reverses its direction. When temperature is increased, the particle will undergo only a hill or a vale for $\phi_{a}<\phi<\pi$ and $\pi<\phi<\phi_{b}$. The current is always zero at $\phi=\pi$. Because of the numerical trouble near $\phi=1.0 \pi$ and low temperature, the numerical results at $\phi=1.0 \pi$ and low temperature meet a little error. However, from the effective potential (Eq. (9)), It is easy to find that the effective potential is symmetric and the current is zero at $\phi=1.0 \pi$. So the line at $\phi=\pi$ should be vertical.

The current $J$ as a function of temperature $T$ is shown in Fig. 3 for different values of $\phi$. When $T \rightarrow 0$, the particle cannot pass the barrier, so the current $J$ tends to zero. When $T \rightarrow \infty$, the thermal noise is very large, so the ratchet effect disappears and the current $J$ approaches zero, also. There exist optimized temperatures at which the current $J$ takes its maximal or minimal values. When $\phi=0.2 \pi\left(0<\phi<\phi_{a}\right)$ and $1.8 \pi\left(\phi_{b}<\phi<2 \pi\right)$, current reversals may occur on increasing temperature. The current is always positive and negative for $\phi=0.7 \pi\left(\phi_{a}<\phi<\pi\right)$ and $1.4 \pi\left(\pi<\phi<\phi_{b}\right)$, respectively. It is easy to see from the Figs. 2 and 3 that the current on increasing temperature may reverse its direction for $0<\phi<\phi_{a}$ and $\phi_{b}<\phi<2 \pi$ and is always positive at all temperatures for $\phi_{a}<\phi<\pi$ or negative for $\pi<\phi<\phi_{b}$.

In order to illustrate the current (see Fig. 3) in detail, the effective potential $U_{\text {eff }}(x)$ along the $x$ coordinate is plotted in Fig. 4. From the previous works $s^{5}-11$, we can obtain that there is no current when the effective potential is symmetric. When the right of the potential in a period is steeper than the left, the current is positive. The current is negative when the left is steeper. In Fig $4(\mathrm{a})(\phi=0.2 \pi)$, the left is steeper than the right (inducing a positive current) at low temperatures. However, when temperature is increased, the right may be steeper than the left (inducing a negative current) at high temperatures. For the case $\phi=1.8 \pi$ (Fig. 4(d)), the current is negative at low temperatures and positive at high temperatures. Therefore, the current can change its direction on increasing temperature for these two cases $(\phi=0.2 \pi$ and $1.8 \pi)$. In Fig. $4(\mathrm{~b})(\phi=0.7 \pi)$, the right is always steeper than the left, so the current is always positive. The left is always steeper than the right at $\phi=1.4 \pi$ (Fig. 4 (c)), so the current is always negative. In these two cases, no current reversal occurs.

Figure 5 shows the current $J$ as a function of the phase shift $\phi$. There is no net current at $\phi=0, \pi$ and $2 \pi$ for all temperatures. There are two peaks and two vales in the curve 
on increasing the phase shift at $T=0.2$ (low temperature). The current can change its direction even three times when the phase shift is changed. At high temperature $(T=0.5)$, only one peak and one vale appear and the current reverses its direction at $\phi=\pi$.

\section{CONCLUDING REMARKS}

In present work, we study the transport of a Brownian particle moving along the axis of three-dimensional periodic tube in the presence of a temporally symmetric unbiased force and a symmetric potential. The movement equation can be described by the Fick-Jacobs equation which is derived from 3D (or 2D) Smoluchowski equation after elimination of $y$ and $z$ coordinates. Reduction of the coordinates may induce the appearance of an entropic barrier and the effective diffusion coefficient. The current is achieved by solving one-dimensional Fick-Jacobs equation.

It is found that the phase shift between the tube shape and the potential may induce a net current even when the potential, the tube shape and the external force are symmetric. The symmetry of the effective potential can be broken by changing temperature or the phase shift. When $0<\phi<\phi_{a}$ and $\phi_{b}<\phi<2 \pi$, current reversal may occur on increasing temperature. However, the current is always positive for $\phi_{a}<\phi<\pi$ or negative for $\pi<\phi<\phi_{b}$. No current occurs at $\phi=0, \pi$ and $2 \pi$ for all temperatures. At low temperatures the current may reverse its direction three times on increasing the phase shift. At high temperatures the current changes its direction one time at $\phi=\pi$.

Our tube model is to describe molecular motors, especially for kinesins and dyneins along microtubule. The potential describes the track of the motor. The external force $F(t)$ depicts the stroke force due to ATP hydrolyzing. The shape of the tube describes the structure of the motor. The phase shift between the tube shape and the potential may induce a net current. Our results can explain current reversals in molecular motors. Several biological molecular motors, for instance kinesin and non-claret disjunctions, belonging to the same superfamily of motor proteins move towards opposite ends of the microtubule ${ }^{7}$. This can be explained by current reversals

The results we have presented have broad applications in many processes ${ }^{12}$, such as molecular motors movement through the microtubule in the absence of any net macroscopic forces ${ }^{17}$, ion transport through ion channels $\frac{18}{}$, motion of polymers subjected to rigid 
constraints $^{19}$, drug release ${ }^{20}$ and polymer crystallization ${ }^{21}$. In these systems, a directedtransport with entropic or energy barriers can be obtained in the absence of any net macroscopic forces or in the presence of the unbiased forces.

\section{ACKNOWLEDGMENTS}

The authors thank Prof. Masahiro NAKANO for helpful discussions. The work is supported by the National Natural Science Foundation of China under Grant No. 30600122 and GuangDong Provincial Natural Science Foundation under Grant No. 06025073.

* Email: aibq@scnu.edu.cn

1 D. Keller and C. Bustamante, Biophysical Journal 78, 541 (2000).

2 C. Bustamante, D. Keller and G. Oster, Acc. Chem. Res. 34, 412 (2001).

3 F. Julicher, A. Ajdari and J. Prost, Rev. Mod. Phys. 69, 1269 (1997).

4 L. P. Faucheux et al., Phys. Rev. Lett. 74 (1995) 1504.

5 J. Luczka, R. Bartussek and P. Hanggi, Europhysics Letters 31 (8), 431-436 (1995).

6 J. L. Mateos, Phys. Rev. Lett. 84, 258 (2000).

7 B. Q. Ai, X. J. Wang, G. T. Liu and L. G. Liu, Phys. Rev. E, 68, 061105 (2003); B. Q. Ai, X. J. Wang, G. T. Liu and L. G. Liu, Phys. Rev. E, 67, 022903 (2003); B. Q. Ai, G. T. Liu, H. Z. Xie, L.G. Liu, Chaos 14(4),957 (2004).

8 M. O. Magnasco, Phys. Rev. Lett. 71, 1477 (1993).

9 P. Hanggi and R. Bartussek, Nonlinear physics of complex system - Current status and Future Trends, 476, Spring, Berlin, (1996), 294.

10 P. Reimann, R. Bartussek, R. Haussler and P. Hanggi, Phys. Lett. A 215, 26 (1994).

11 C. R. Doering, W. Horsthemke and J. Riordan, Phys. Rev. Lett. 72, 2984 (1994).

12 D. Reguera, and J. M. Rubi, Phys. Rev. E 64, 061106 (2001).

13 D. Reguera, G. Schmid, P. S. Burada, J. M. Rubi, P. Reimann, and P. Hanggi, Phys. Rev. Lett 96, 130603 (2006).

14 M. Badoual, F. Julicher and J. Prost, PNAS 99, 6696 (2002)

15 R. Zwanzig, J. Phys. Chem. 96, 3926 (1992). 
16 M. H. Jacobs, Diffusion Processes (Springer, New York, 1967).

17 J. D. Huang, S. T. Brady, B. W. Richards, D. Stenolen, J. H. Resau, N. G. Copeland and N. A. Jenkins, Nature 397, 204 (1999).

18 P. Gates, K. Cooper, J. Rae, and R. Eisenberg, Prog. Biophys. Mol. Biol. 53, 153 (1990).

19 M. Fixman, J. Chem. Phys. 69, 1527 (1978).

20 R. A. Seigel, J. Controlled Release 69, 109 (2000).

21 J. P. K. Doye and D. Frenkel, J. Chem. Phys. 110, 7073 (1999). 


\section{CAPTION LIST}

FIG. 1. The upper is schematic diagram of tube with periodicity $L$. The shape is described by the radius of the tube $\omega(x)=a \sin \left(\frac{2 \pi x}{L}+\phi\right)+b$. The bottom is the potential along the $x$ coordinate with periodicity $L, U(x)=Q \sin \left(\frac{2 \pi x}{L}\right)$.

FIG. 2. Current contours on $T-\phi(\pi)$ plane at $Q=0.5, a=\frac{1}{2 \pi}, b=\frac{1.5}{2 \pi}, \alpha=1 / 2$ and $F=0.5$. The current is negative in area $\mathrm{A}$ and $\mathrm{C}$, positive in area $\mathrm{B}$ and $\mathrm{D}$. The solid line denotes zero current. $\phi_{a}(0.3817)$ and $\phi_{b}(1.6035)$ are the intersectant point between the $\phi$-axis and the zero-current line near area $\mathrm{A}$ and $\mathrm{D}$, respectively.

FIG. 3. Current $J$ vs temperature $T$ for different values of $\phi$ at $Q=0.5, a=\frac{1}{2 \pi}, b=\frac{1.5}{2 \pi}$, $\alpha=1 / 2$ and $F_{0}=0.5$.

FIG. 4. The effective potential $U_{\text {eff }}(x)$ along the $x$ coordinate for different values of $T$. From the bottom to the upper the temperature is increased. (a) $\phi=0.2 \pi$; (b) $\phi=0.7 \pi$; $(\mathrm{c}) \phi=1.4 \pi ;(\mathrm{d}) \phi=1.8 \pi$.

FIG. 5. Current $J$ vs the phase shift $\phi$ for different temperature at $Q=0.5, a=\frac{1}{2 \pi}$, $b=\frac{1.5}{2 \pi}, \alpha=1 / 2$ and $F_{0}=0.5$. 


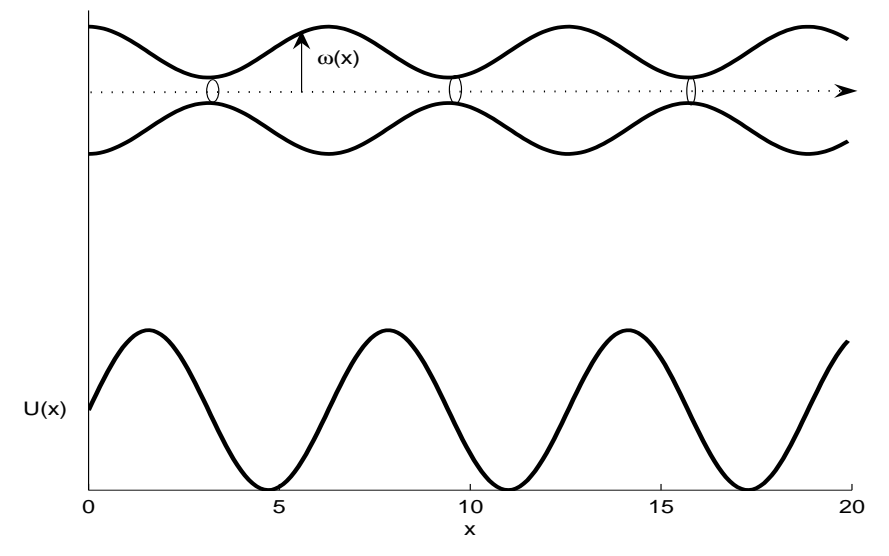

FIG. 1: 


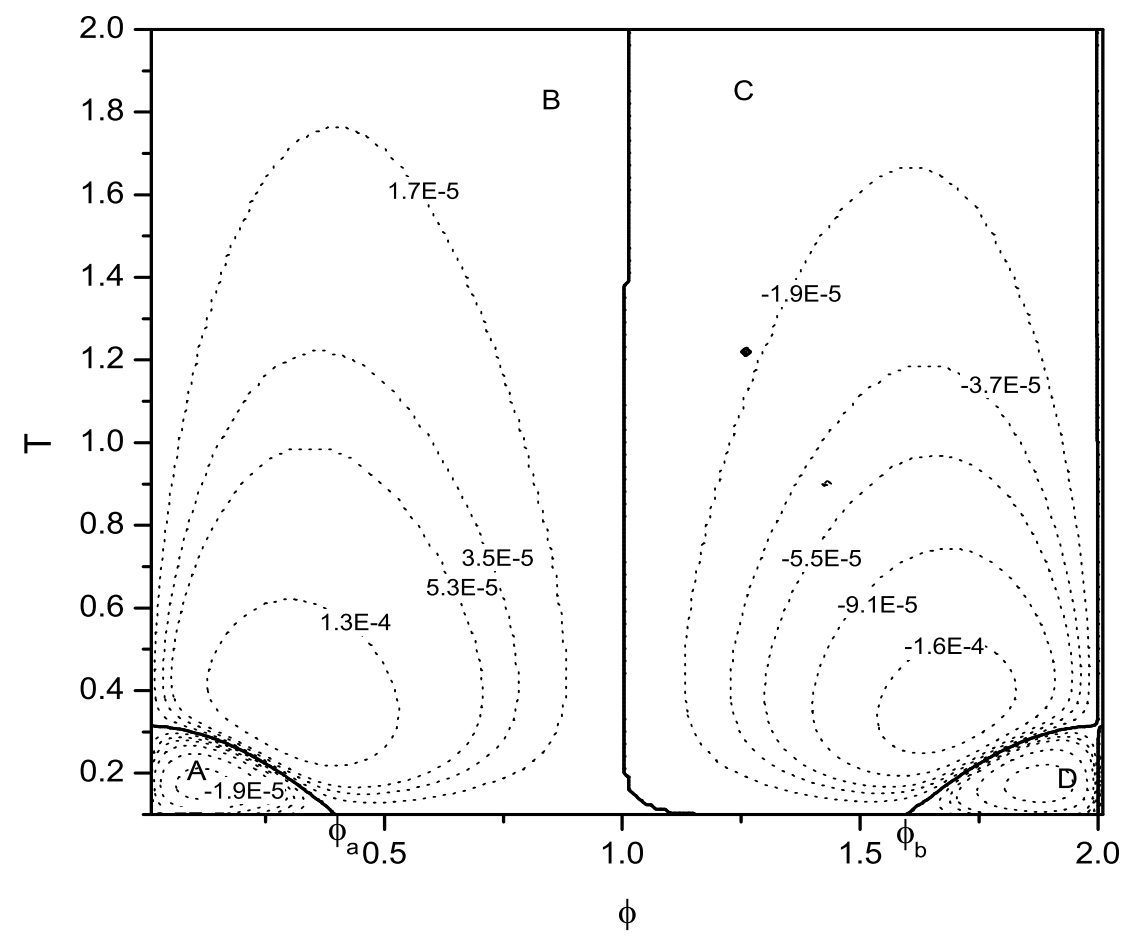

FIG. 2: 


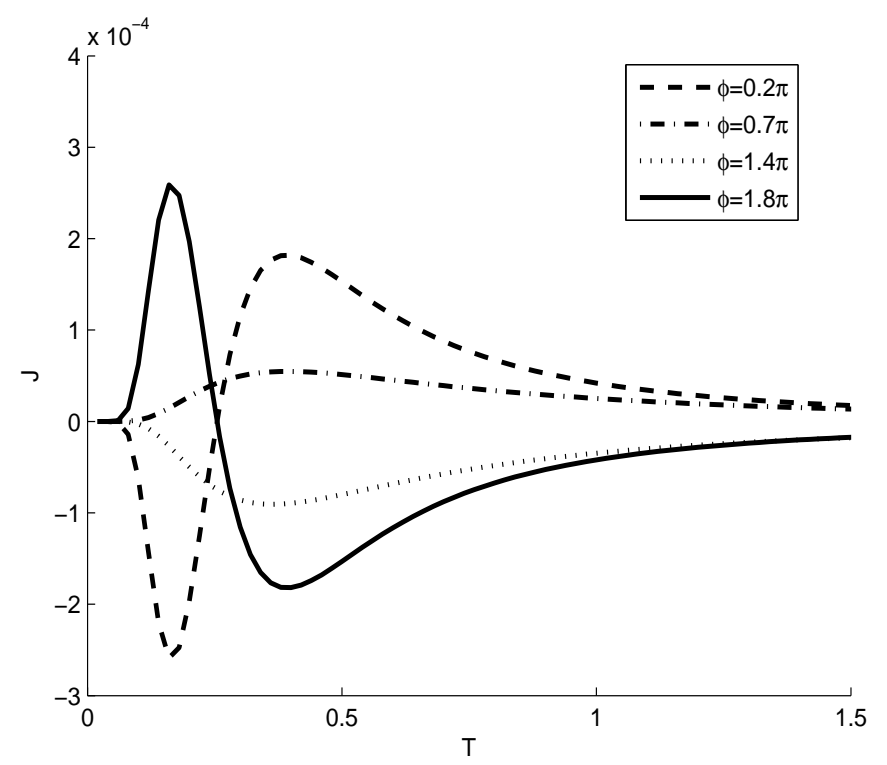

FIG. 3: 

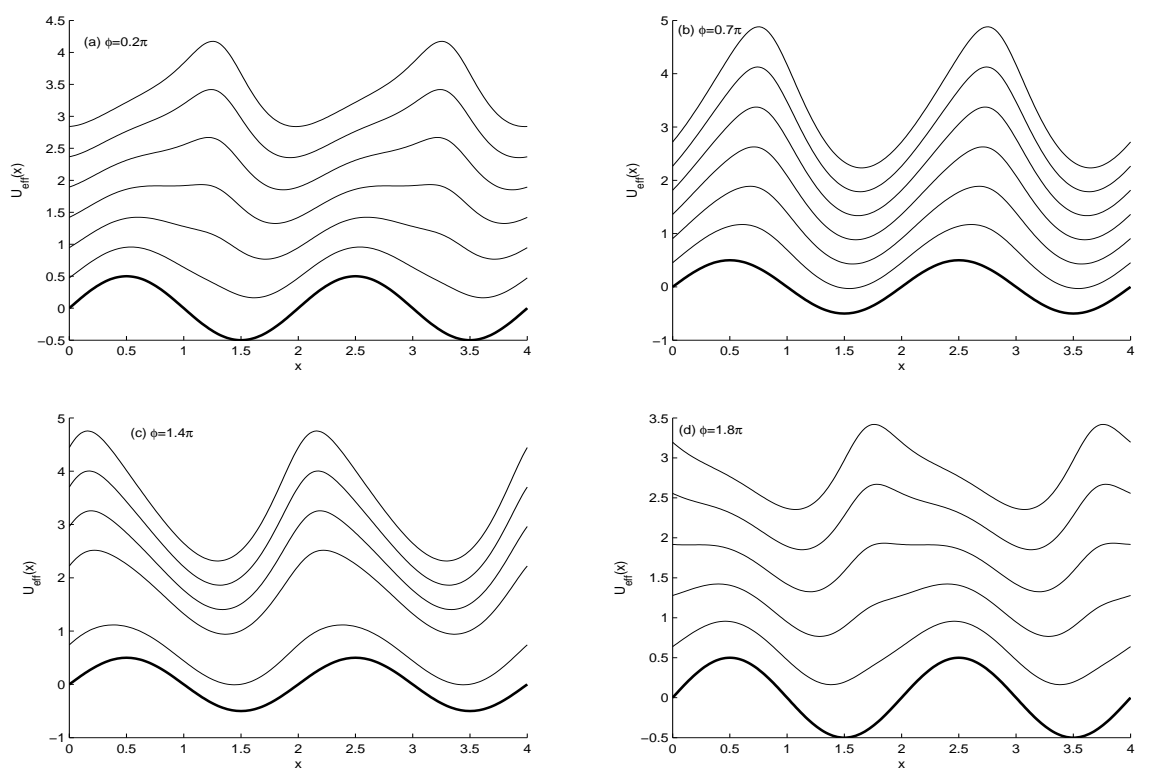

FIG. 4: 


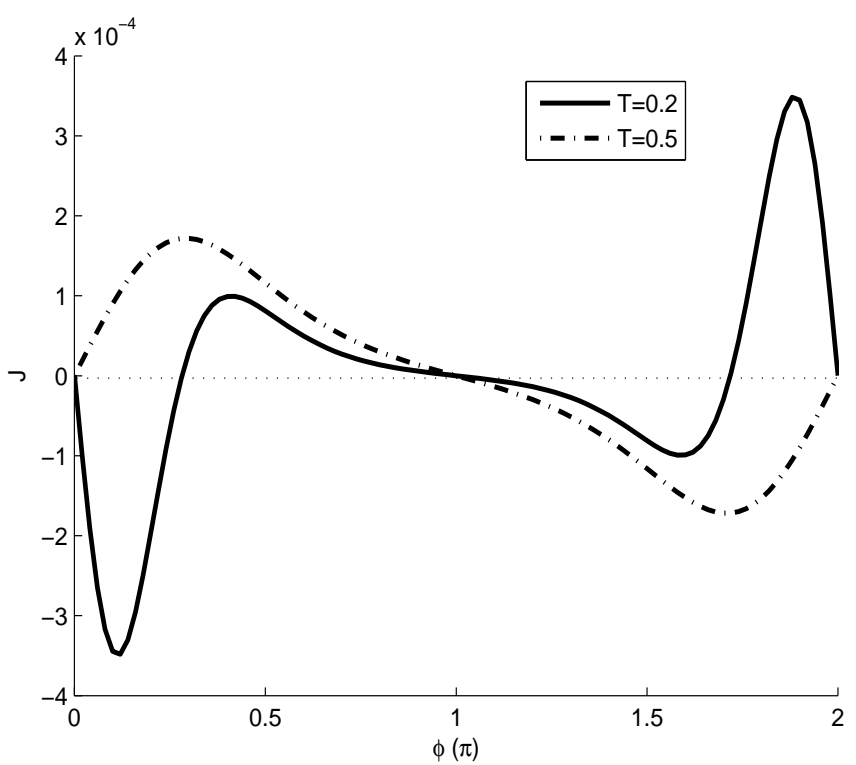

FIG. 5: 